\title{
A Young Girl with Barely Symptomatic Congenital Mitral Valve Disease
}

\author{
Gobinda Kanti Paul', Muhammad Towhidul Ahsan Khan ${ }^{1}$, Mohammad Abdus Sattar Bhuiyan ${ }^{1}$, \\ Mohammad Alwalid Sharker ${ }^{1}$, Jahir Mohammed Sharif ${ }^{1}$, Nusrat Jahan ${ }^{1}$, Koushik Bhowmick ${ }^{1}$, \\ Md. Shamim Chowdhury ${ }^{2}$
}

${ }^{1}$ Department of Cardiology, Mymensingh Medical College and Hospital, Mymensingh, ${ }^{2}$ National Heart Foundation Hospital \& Research Institute, Dhaka.

Keywords:
Congenital heart
disease, mitral
valve, double
orifice mitral
valve.

\begin{abstract}
:
\end{abstract}
Congenital mitral valve disease or malformation may be isolated or associated with other cardiac anomalies. Mitral valve may affect its different segments during development. This anomaly is due to mitral valve with a single fibrous annulus with two orifices (Double orifices mitral valve) that open into the left ventricle. It may be associated with stenosis and or mitral regurgitation. Here we describe a 17 years young girl with palpitation and shortness of breath on moderate to severe exertion. Examination of the cardiovascular system, chest including precordium revealed no abnormality. She is acyanotic and there is no clubbing. On Trans thoracic Echocardiogram isolated double orifice mitral valve was found with no other associated congenital anomalies.

(Cardiovasc j 2021; 14(1): 79-81)

\section{Introduction:}

Double orifice mitral valve (DOMV) is a rare congenital anomaly of the subvalvular mitral valve apparatus (Chordae tendinae and papillary muscles) consisting of an accessory bridge of fibrous tissue, which partially or completely divides the mitral valve into two orifices. ${ }^{1}$ Anatomically and functionally, the mitral cusps are essentially normal in most cases, but may be associated with mitral stenosis or regurgitation. DOMV rarely occurs as an isolated anomaly, but may be associated with other cardiac anomalies such as atrioventricular defects, coarctation of the aorta, bicuspid aortic valve, ventricular septal defect, patent ductus arteriosus, hypoplastic left heart syndrome etc. ${ }^{1}$ The prevalence of isolated DOMV in adulthood is not known.

\section{Case Report}

17 years young girl has got palpitation \& shortness of breath on moderate to severe exertion for 1 month. There is no cough, fever. She has got no history suggestive of rheumatic fever. On general examination, cyanosis \& clubbing was absent. Examination of cardiovascular system, chest including precordium revealed no abnormality. All the routine blood tests were normal. ECG \& chest X-ray was also normal. Trans thoracic Echocardiography, performed with Philips Affinity 70 , revealed double orifice mitral valve but no other abnormalities and associated any other congenital anomalies. In subsequent follow up, non-specific complaints from the patient and advised for taking Propranolol and at present she feeling better. The patient was instructed to consult with us if any symptom evolves.

Address of Correspondence: Dr. Gobinda Kanti Paul, Dept. of Cardiology, MMC\&H, Mymensingh, Bangladesh. Emaildrgobinda@yahoo.com.

- 2020 authors; licensed and published by International Society of Cardiovascular Ultrasound, Bangladesh Chapter and Bangladesh Society of Geriatric Cardiology. This is an Open Access article distributed under the terms of the CC BY NC 4.0 (https://creativecommons.org/licenses/by-nc/4.0) 


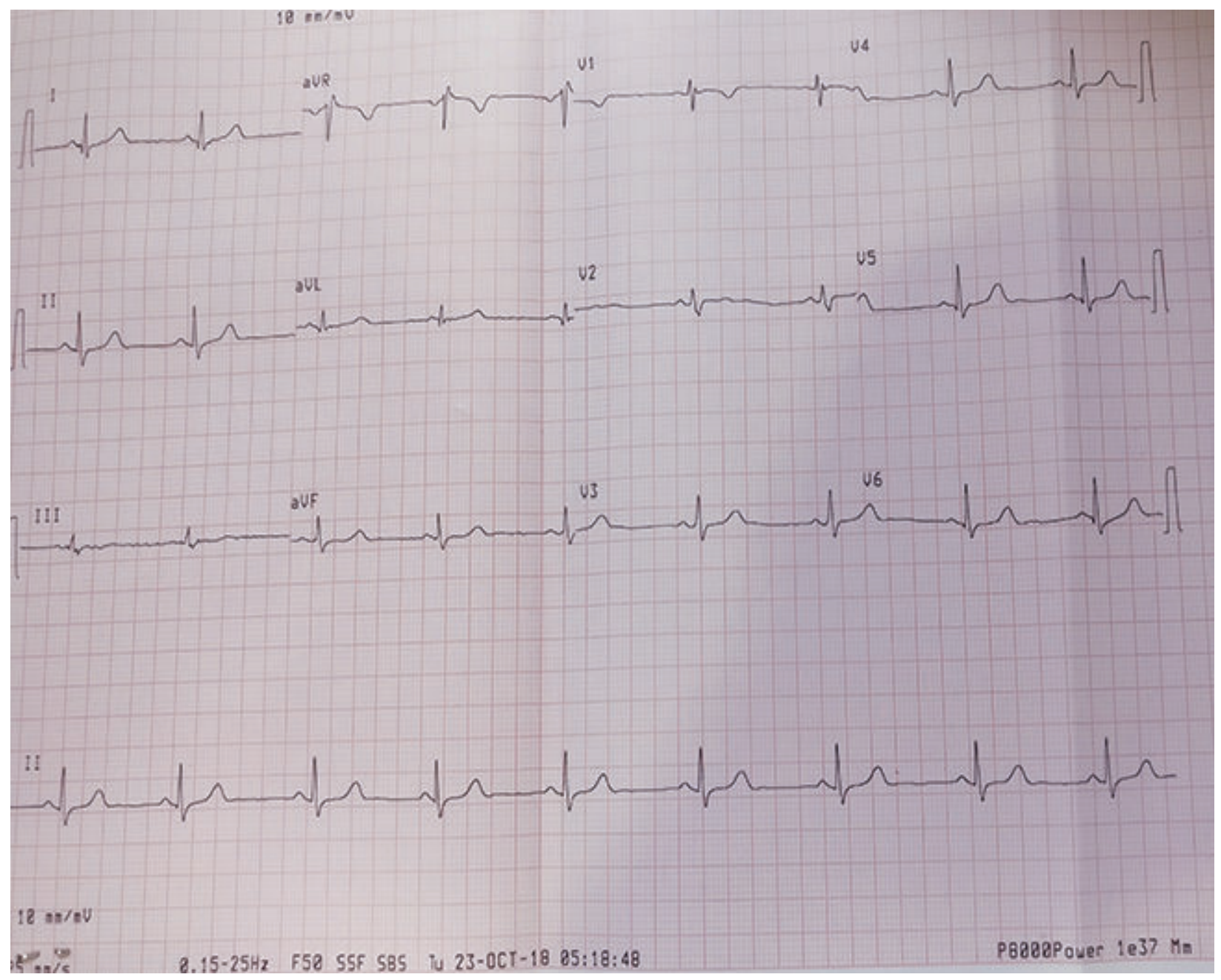

Fig.-1: 12 lead ECG showing normal tracing.

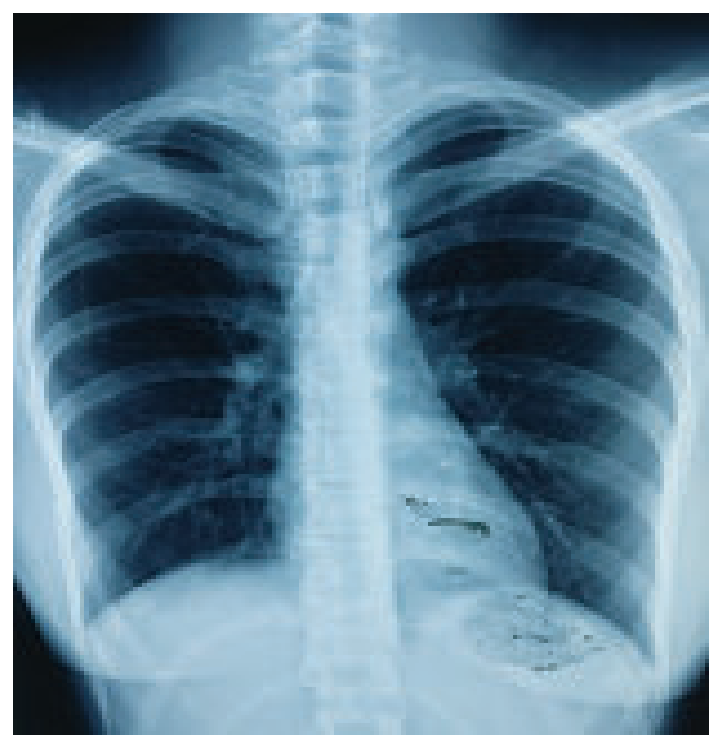

Fig.-2: Chest $X$-ray $P A$ view shows normal findings.

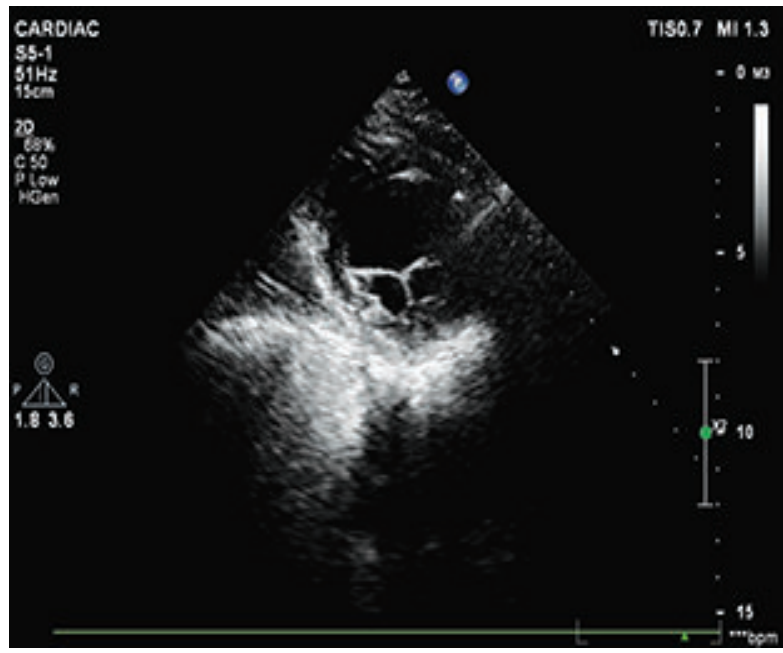

Fig.-3: Parasternal short axis (PASX) view shows, two mitral orifices separated by fibrous band (Owl's eye appearance). 


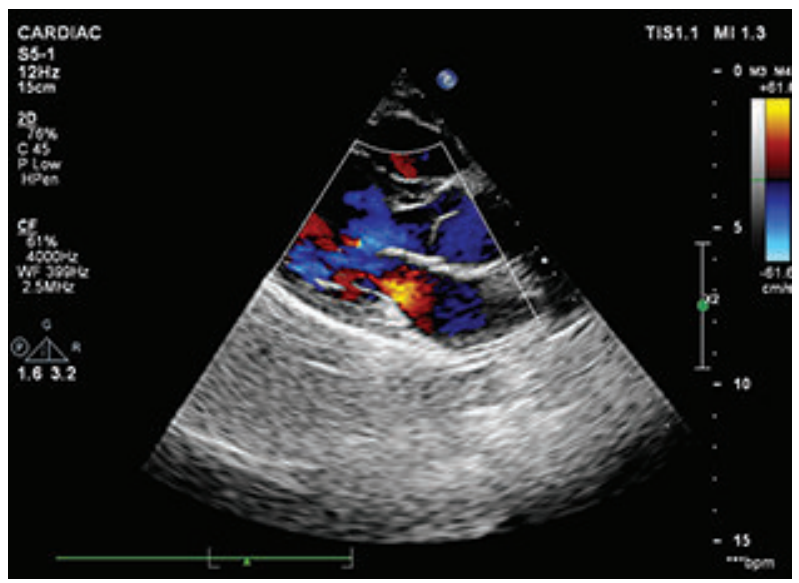

Fig.-4: 2D guided CFM, PLAX view shows normal flow pattern, across the mitral \& aortic valve.

\section{Discussion:}

Double orifice mitral valve is an uncommon congenital mitral valve defect that was first described by William Smith Greenfield in $1876 .{ }^{2}$ Recognition of double orifice mitral valve and awareness of the anatomic variations are important to achieve good therapeutic results. DOMV is an extremely rare congenital anomaly, classified into three categories: complete bridge (15\%), incomplete bridge and hole type (85\%). ${ }^{3}$ As an isolated DOMV without functional abnormalities of the mitral valve, usually causes no symptoms. In a large autopsy series of patients with congenital heart disease, $1 \%$ had a DOMV, which in the majority of cases was associated with other cardiac anomalies. ${ }^{4}$
Our case presents as a mildly symptomatic and isolated DOMV without any stenosis or regurgitation. We have to diagnose only by transthoracic echocardiography. Some time needed 3D echo for better evaluation, especially in complex cases. Treatment is only necessary if significant mitral stenosis or mitral regurgitation is present.

\section{Conclusion:}

Echocardiography is the best way for comprehensive diagnosis of DOMV with anatomical and functional assessment. In the presence of DOMV concomitant cardiac anomalies and valvular stenosis or regurgitation must be excluded. If an isolated DOMV with no functional abnormalities is present, usually no further follow-up is necessary.

\section{Conflict of Interest - None.}

\section{References:}

1. LINKA A. Double orifice mitral valve. Heart. 2000; 84(3): 244-244. DOI:10.1136/heart.84.3.244

2. Greenfield W. Double mitral valve. Trans Pathol Soc (London). 1876; 27: 128-129.

3. Trowitzsch E, Bano-Rodrigo A, Burger B, Colan S, Sanders S. Two-dimensional echocardiographic findings in double orifice mitral valve. J Am Coll Cardiol. 1985;6(2): 383-387. DOI:10.1016/s0735-1097(85) 80176-5

4. Baño-Rodrigo A, Van Praagh S, Trowitzsch E, Van Praagh R. Double-orifice mitral valve: A study of 27 postmortem cases with developmental, diagnostic and surgical considerations. Am J Cardiol. 1988; 61(1): 152160. DOI:10.1016/0002-9149(88)91322-7 